\title{
VORTEX-INDUCED EXTINCTION BEHAVIOR IN METHANOL GASEOUS FLAMES: A COMPARISON WITH QUASI-STEADY EXTINCTION
}

\author{
VITO S. SANTORO, DIMITRIOS C. KYRITSIS, AMABLE LIÑÁN AND ALESSANDRO GOMEZ \\ Yale Center for Combustion Studies \\ Yale University \\ New Haven CT 06520-7682, USA
}

\begin{abstract}
Using a combination of HCHO planar laser-induced fluorescence and laser Doppler velocimetry measurements, the extinction behavior of methanol counterflow diffusion flames was examined experimentally under conditions in which the extinction was brought about by a vortex generated on the oxidizer side. Comparisons were made with quasi-steady extinction results for the same flames. It was found that the flames can withstand instantaneous strain rates as much as two-and-a-half times larger than the quasisteady ones. The finding was rationalized phenomenologically by comparing the characteristic times of the problem, that is, the mechanical time, the chemical time, and the vortex turnover time. Specifically, estimates of these times yielded the following ordering: $\tau_{\mathrm{ch}}<\tau_{\mathrm{vort}}<\tau_{\mathrm{m}}$. As a result, the vortex introduced an unsteady effect in the outer diffusive-convective layer of the flame, while the inner reactive-diffusive layer behaved in a quasi-steady manner. Consequently, the flame was subject to a damped strain rate through the outer layer. Results from a simple analytical model showed that the difference between vortexinduced extinction and quasi-steady extinction was much more modest in terms of instantaneous scalar dissipation rate or Damköhler number. Furthermore, the temporal history of the strain rate was found to be necessary to determine the effective strain rate felt by the flame. Implications of these findings for turbulent diffusion flame modeling by the flamelet approach are discussed.
\end{abstract}

\section{Introduction}

The presence of vortical structures in turbulent diffusion flames and the use of flamelet models suggest a laminar vortex interacting with a counterflow diffusion flame as a convenient system in which time-dependence and curvature effects can be independently examined [1]. Our objective was to study such a well-defined system experimentally and provide information to models of turbulent combustion. Two classes of investigations are relevant to the present work: one deals with vortex-flame interaction, and the other is restricted only to time-dependent perturbations.

The first class was ushered in by some analytical work $[2,3]$, followed by direct numerical simulation of flame-vortex interaction with simplified kinetics [1]. Experimentally, the interaction of a vortex with a laminar flame was introduced first in a premixed configuration [e.g., 4,5]. In diffusion flames, the first qualitative experimental investigation on hydrogen/ air systems was reported in Ref. [6], followed by computational modeling of the same system in Ref. [7], and by a study of its extinction behavior in Ref. [8]. In the latter, experimental results on the flamesurface evolution were interpreted in terms of a stretch rate and compared to extinction strain rates obtained from quasi-steady asymptotic analysis, with the justification that the flame-response time was fast enough for the flame to be able to adapt rapidly to transient conditions. The last contribution from the same group showed a variety of morphologies ensuing flame-vortex interaction for the same hydrogen/air flames [9]. A new type of extinction, an annular one, that is probably peculiar to the hydrogen/ air flames, was observed in Ref. [10] and attributed to preferential diffusion and curvature.

The second class of relevant studies is that on transient effects in one-dimensional counterflow diffusion flames. If curvature effects can be neglected, the vortex-flame interaction can be described as an unsteady perturbation of the one-dimensional field. Transient effects have been studied for some time and were first introduced in a partly analytical, partly experimental investigation [11]. In subsequent years, a number of computational [12-15] and analytical [16-18] studies followed. Most pertinent is a systematic analysis of flame extinction in Ref. [19] that was in part corroborated in Ref. [20]. In all of these studies, the flame was typically subjected to either a periodic perturbation or a step change in velocity profile at the boundary, and its response was studied as a function of frequency and amplitude of the disturbance. The common feature emerging from these studies is that the flame response to rapid changes in strain rate is attenuated as the timescale of the perturbation decreases. As a result, the flame can momentarily sustain higher strain rates than its 


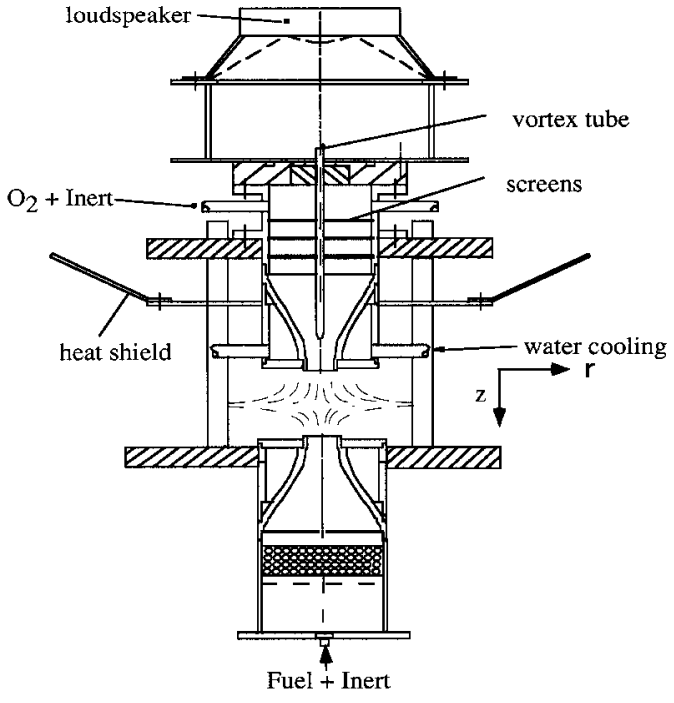

FIG. 1. Burner configuration.

quasi-steady extinction value without extinguishing. The most recent study on transient effects stresses the difference in chemical response of relatively slow reactants (e.g., $\mathrm{CO}$ and $\mathrm{NO}_{x}$ ) as compared to relatively fast intermediates (e.g., $\mathrm{OH}$ and $\mathrm{H}$ ) [21].

In the present investigation, we examined extinction of gaseous counterflow diffusion flames of methanol interacting with a vortex and contrasted it with the same phenomenon in quasi-steady flames for different flame compositions. Unlike all other studies on vortex-flame interaction in diffusion flames [6-10], we focused on an alcohol, specifically methanol, to eliminate peculiarities of hydrogen/air flames associated with preferential diffusion and Lewis number effects. Here, we provide a set of experimental data on the peak extinction strain rates sustainable by the flames. The results were interpreted in terms of a simple analytical model that showed that the time history of the strain rate was important. We conclude by discussing the implications of our findings to flamelet modeling of turbulent flames.

\section{Experimental Apparatus}

An axisymmetric counterflow diffusion flame was established in a vertical configuration, as shown in Fig. 1, with the oxidizer being fed from one side, and methanol and inert fed from the other. Both sides of the burner terminated in a contraction, which was contoured following a well-established wind-tunnel design practice, which together with the use of an assortment of meshes and screens on the flow path, optimized the uniformity of the axial velocity in the radial direction. The exit diameter of the two nozzles was $12.5 \mathrm{~mm}$, and the separation distance between them was kept constant at $13 \mathrm{~mm}$. Flanges were fitted to the tube outlets in order to prevent buoyancy effects from altering the flatness of the flame around the burner centerline. Cooled water was circulated through the upper flange to keep it at the desired temperature. Methanol was used as a fuel. It was prevaporized by bubbling $\mathrm{He}$ through a liquid methanol bath.

The steady flame was perturbed by periodically generated laminar toroidal vortices. To that end, a suitably synthesized voltage function was applied across the loudspeaker, causing the latter to force air impulsively through a $1.5 \mathrm{~mm}$ tube, similarly to the system described in Ref. [6]. The tube was positioned at a distance from the outlet of the oxidizer nozzle in order to minimize its effect on the axial velocity profile in the unperturbed flame. Furthermore, care was required in the design of the voltage function to avoid ringing and overheating of the loudspeaker coil, which would have resulted in the formation of multiple vortices or in permanent damage of the device. The vortices were visualized using planar light scattering of submicron $\mathrm{TiO}_{2}$ particles that were produced by hydrolysis of $\mathrm{TiCl}_{4}$. This technique was used to measure vortex dimensions and translational velocity and to assess the vortex laminarity and the repeatability of the generation scheme.

To monitor the flame dynamics under vortex excitation, planar-induced fluorescence of naturally occurring formaldehyde, HCHO, was used. Formaldehyde, in fact, is an intermediate that is present in the dominant oxidation path of methanol: $\mathrm{CH}_{3} \mathrm{OH}$ $\rightarrow \mathrm{HCHOH} \rightarrow \mathrm{HCHO} \rightarrow \mathrm{HCO} \rightarrow \mathrm{CO}$. Furthermore, for methanol flames, the pyrolysis of $\mathrm{HCHOH}$ to HCHO represents the critical step for extinction [22]. The suitability of HCHO laser-induced fluorescence for the present purposes was confirmed by the results of a computational model of a steadystate counterflow diffusion flame, with detailed kinetics and transport, that indicated that for gaseous methanol flames the peak of heat release corresponds to the location where the formaldehyde concentration precipitously drops [23]. These results suggest that formaldehyde can indeed be used as a complementary marker for methanol flames, since this marker concentration decreases dramatically as the heat-release rate rises.

To visualize formaldehyde, the third harmonic of a Nd:YAG laser with $120 \mathrm{~mJ} /$ pulse of energy at 355 $\mathrm{nm}$ was used to excite the $4_{0}^{1}$ transition in the $\tilde{A}^{2} A_{2}$ $\leftarrow \tilde{X}^{1} A_{1}$ band of HCHO, as previously described in Ref. [23]. The resulting signal was detected using a gated single-stage image intensifier coupled to a charge-coupled device (Santa Barbara Instrument Group ST6B). A narrow bandpass interference filter at $415 \mathrm{~nm}$ with a full width at half maximum of 10 $\mathrm{nm}$ was used to reject flame luminescence and other 


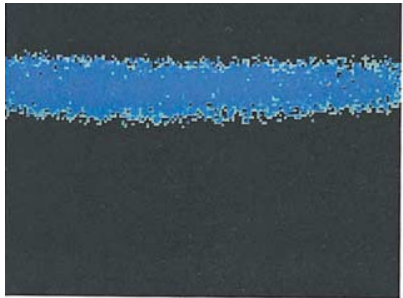

a)

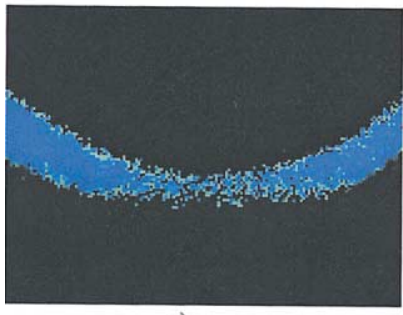

c)

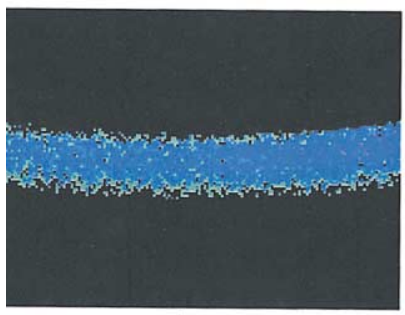

e)

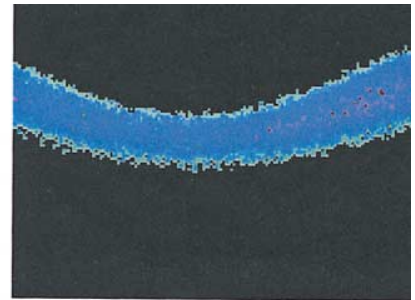

b)

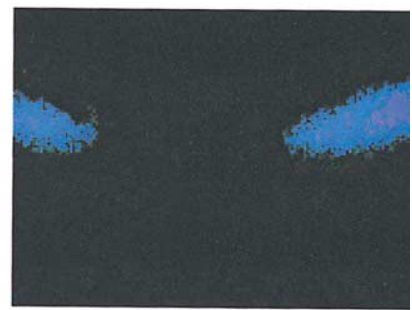

d)

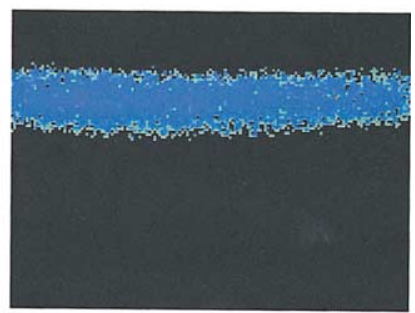

f)
FIG. 2. Sequence of PLIF images during vortex-flame interaction for a prototypical flame. The times elapsed after vortex generation are (a) $t=0 \mathrm{~ms}$, (b) $t=18 \mathrm{~ms}$, (c) $t=$ $24 \mathrm{~ms}$, (d) $t=26 \mathrm{~ms}$, (e) $t=35 \mathrm{~ms}$, (f) $t=85 \mathrm{~ms}$. interference. Both planar diagnostic techniques were synchronized with the signal generator that produced the vortex, using a variable time delay. In this way the evolution of the interaction between the vortex and flame could be monitored at any desired instant in time.

The fluorescence approach is straightforward to implement because it does not involve a dye laser, but it is not optimal because the YAG third harmonic excites the tail of the absorption band of formaldehyde that peaks at $353.2 \mathrm{~nm}$. No background subtraction from polycyclic aromatic hydrocarbons could be applied by looking at the off-resonance signal. Nevertheless, their interference is not believed to be significant.

Planar diagnostic techniques were complemented by measurements of gas velocity and droplet diameter by a commercial phase doppler anemometer. The velocity of the gas was determined using seed particles of $\mathrm{Al}_{2} \mathrm{O}_{3}$, with a diameter of $1.5 \mu \mathrm{m}$. The single-point velocities were phase averaged and used to evaluate the strain rate along the centerline of the flame and the major vortex parameters, such as diameter, core-to-core distance, translational velocity, rotational velocity, and circulation. The $95 \%$ confidence interval was estimated at $+/-0.02$ of the mean. It was computed with a conservative assumption of $1 \%$ bias error (based on manufacturer specifications) and a precision limit of the mean of $1.5 \%$.

\section{Results and Discussion}

A steady flat flame was established in the middle of the two nozzles and subsequently perturbed by vortices generated on the oxidizer side. Under vortex excitation, localized wrinkling in the vicinity of the centerline was observed, which for sufficiently strong vortices, yielded local extinction with the development of a hole in the middle of the flame. In Fig. 2, a selected sequence of single-shot planar laser-induced fluorescence (PLIF) images is presented for a typical gaseous flame. These images show critical aspects of the interaction. Eighteen ms after being generated, the vortex approached the flame and wrinkled it. The interaction evolved, with the flame moving toward the fuel side because of the increased momentum from the opposite side, 


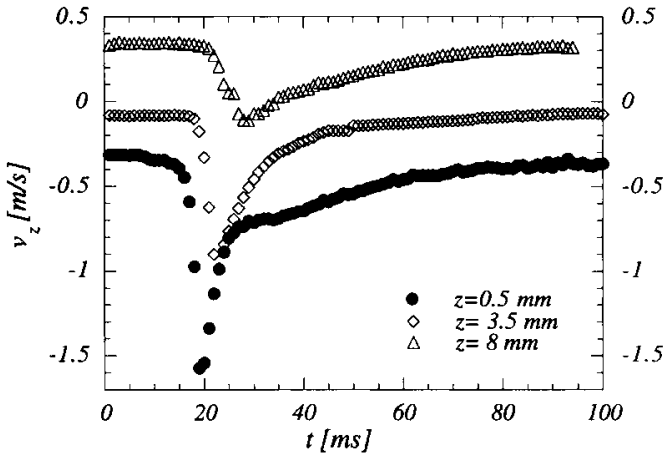

FIG. 3. Phase-averaged axial velocity measurements as a function of time at three locations along the flame.

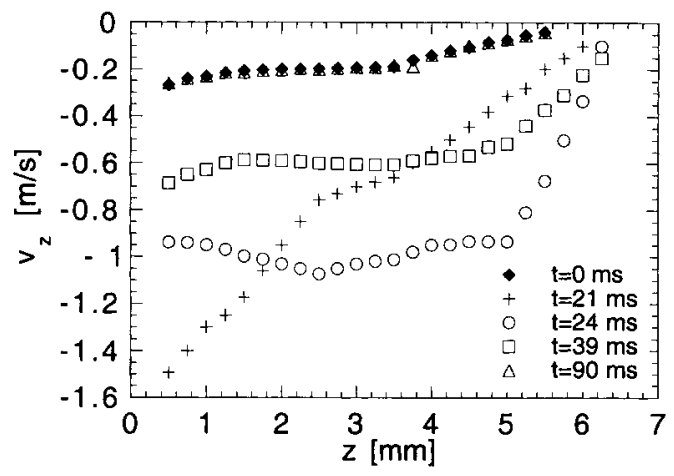

FIG. 4. Phase-averaged axial velocity measurements as a function of the axial coordinate at selected times.

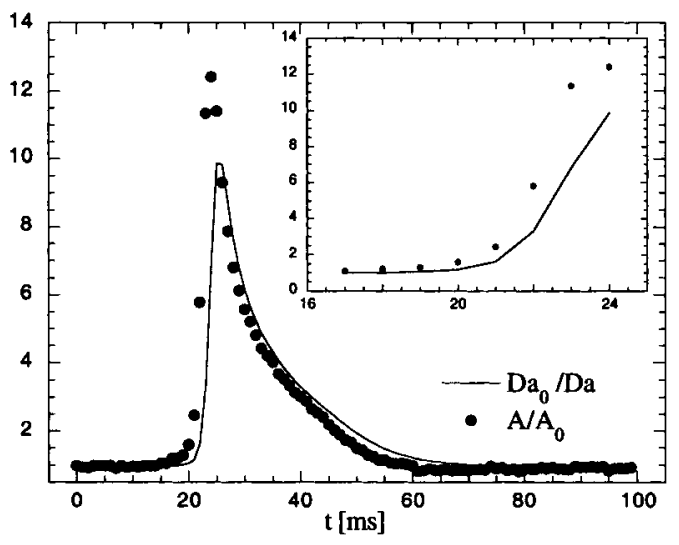

FIG. 5. Non-dimensional experimental strain rate near the flame as a function of time (symbols). Damköhler number, normalized with respect to the baseline value, as a function of time, as estimated from a thermal-diffusive model. Inset: magnification of the onset of the perturbation. and the formaldehyde layer becoming thinner because of the momentary increase in strain rate. At $t$ $=24 \mathrm{~ms}$, the flame was not able to sustain the high strain rate locally, and a hole developed. One millisecond later, the hole diameter already measured 4 $\mathrm{mm}$ in diameter; the propagation of the extinction front in the radial direction was helped by the radial flow in the outer direction. The extinction front stopped when the hole reached a diameter of $6 \mathrm{~mm}$. After the passage of the vortex, the now annular flame closed in and re-established a flat and uniform diffusion flame, moving back to the original position, as strain rate and momentum slowly decreased.

Once the conditions at which extinction occurred were established using the PLIF images, the temporal evolution of the strain rates was measured. Under conditions such as those in Fig. 2, the time trace of the axial velocity component at selected locations along the centerline was measured and the data were phase averaged. In Fig. 3, the phase-averaged axial velocity profile is shown as a function of time at three axial positions, $0.5 \mathrm{~mm}, 3.5 \mathrm{~mm}$, and $8 \mathrm{~mm}$, below the oxidizer nozzle. The velocity profile is strongly perturbed by the presence of the vortex. The velocity decreases from a steady-state value to a minimum, and then it recovers the original value, the implication being that no residual effect due to the previous vortex is present when a new one is generated. Using an external reference time provided by the function generator, the velocity measurements could be plotted as a function of the axial position at any instant in time. Fig. 4 presents such data on the oxidizer side, with the axial velocity plotted as a function of the axial coordinate at a few selected times. The gas stagnation plane is displaced by the increase in momentum induced by the vortex; therefore, the particles, upon vortex arrival, can travel further along the centerline, as shown by the rightmost points at $t=24 \mathrm{~ms}$ in Fig. 4 . The strain rate, $\partial v / \partial z$, was evaluated as the slope of the axial velocity across the five rightmost data points in the figure, corresponding to the last millimeter before the flame. The change of this slope in time, non-dimensionalized with respect to its initial value, is shown in Fig. 5. The strain rate increases, reaches a maximum at $t=$ $24 \mathrm{~ms}$ and then relaxes back to the initial value. The combination of velocity measurements and PLIF allowed us to measure the extinction strain rate, that in this particular case, corresponded to the peak value. Comments on the continuous line in Fig. 5 and on the inset are made below.

For a steady counterflow diffusion flame, the strain rate scales as

$$
A=\frac{2 U_{\mathrm{O}_{2}}}{L}\left[1+\frac{U_{\mathrm{F}}}{U_{\mathrm{O}_{2}}}\left(\frac{\rho_{\mathrm{F}}}{\rho_{\mathrm{O}_{2}}}\right)^{1 / 2}\right]
$$

where $\rho_{\mathrm{F}}$ and $\rho_{\mathrm{O}_{2}}$ represent the density of the gases on the fuel side and on the oxidizer side, respectively, $L$ is the separation distance between the two 


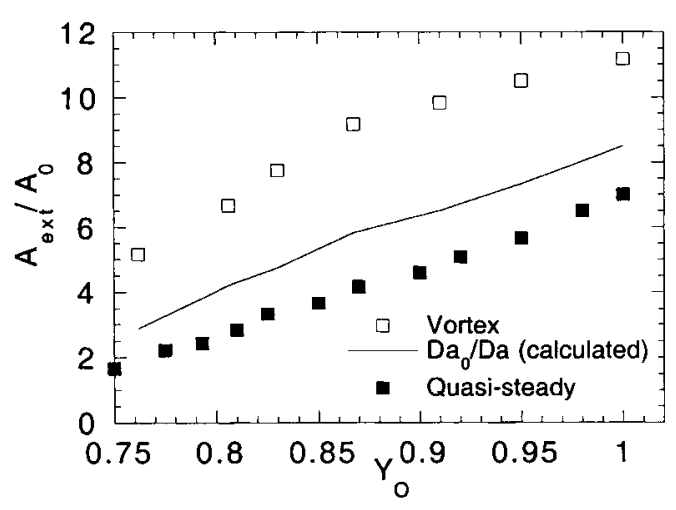

FIG. 6. Comparison of vortex-induced extinction strain rates and quasi-steady extinction values as a function of flame composition. The experimental values are normalized with the baseline strain rate. The continuous line is estimates of normalized Damköhler number based on a thermal-diffusive model.

nozzles, $U_{\mathrm{F}}$ is the axial velocity at the fuel outlet nozzle, and $U_{\mathrm{O}_{2}}$ is the axial velocity at the oxidizer outlet nozzle [24]. The flame strain rate can be changed in different ways by changing any of these parameters. In the present investigation, various flames were studied under vortex excitation. The vortex parameters were kept constant, while the boundary conditions of the flame were changed systematically by keeping constant the baseline strain rate at $55 \mathrm{~s}^{-1}$ and changing the composition of the feed streams. Specifically, the fuel mass fraction was kept constant at 0.67 , whereas the oxidizer mass fraction was changed from unity to 0.75 . The results are reported in Fig. 6. The vortex-induced extinction strain rate is shown with open symbols. Also reported in the same figure are the results of the quasisteady extinction (full symbols), that is, the extinction obtained under the same composition but without vortex excitation. In the latter case, extinction was induced by slowly increasing the mass flux from both sides while balancing momentum to make sure that the flame position did not appreciably change, until extinction was observed. All results were normalized with the baseline strain rate and plotted versus the oxygen mass fraction in the oxidizer stream.

From this graph, a remarkable difference between the quasi-steady extinction strain rate and the vortexinduced one emerges. The $\mathrm{CH}_{3} \mathrm{OH}$ flame was found to be remarkably more robust under time-dependent perturbations, with peak extinction strain rates exceeding the quasi-steady value by as much as a factor of 2.5. A qualitative explanation of the difference between steady-state and vortex-induced extinction can be found by considering the timescales involved in the problem. Specifically one should consider the mechanical time, $\tau_{\mathrm{m}}$, the inverse of the base-line strain rate; the chemical time, $\tau_{\mathrm{ch}}=1$ / $\left(A \beta^{2}\right)$, that can be evaluated from the steady-state extinction curve by assuming a one-step reaction rate, with $\beta$ being the Zeldhovic number that is typically of order 10; and the characteristic vortex turnover time, $t_{\text {vort }}=\pi(d / 2)^{2} / \Gamma$, where $\Gamma$ is the circulation and $d$ is the vortex core diameter, as evaluated from velocity measurements similarly to the approach used in Ref. [4]. The values of the variables needed for this estimate are $\beta \approx 8+12, d=1.2$ $\mathrm{mm}$, and $\Gamma=5 \times 10^{-3} \mathrm{~m}^{2} / \mathrm{s}$, measured experimentally at the exit of the oxidizer nozzle. Thus, the order of magnitude estimate of the characteristic timescales is $\tau_{\mathrm{m}}=A^{-1}=17 \mathrm{~ms}, \tau_{\mathrm{ch}} \approx 0.2+0.3$ $\mathrm{ms}, \tau_{\text {vortex }}=2.4 \mathrm{~ms}$, that yields the following ordering: $\tau_{\mathrm{ch}}<\tau_{\text {vort }}<\tau_{\mathrm{m}}$. As a result, the vortex introduces an unsteady effect in the outer diffusive-convective layer, while the inner reactive-diffusive layer behaves in a quasi-steady manner, since the characteristic chemical time is much smaller than the characteristic unsteady time. Consequently, even though the instantaneous strain rate is much larger than the quasi-steady extinction strain rate, the flame is subject to a strain rate damped through the outer layer.

The phenomenological explanation given above can be substantiated using a simple analytical model that takes into account the variation of the thickness of the mixing layer with time and uses the constant density approximation, as in Ref. [25]. The extinction of the diffusion flame occurs when, with increasing strain rates, the mass burning rate of fuel per unit flame surface increases above a critical value. This phenomenon can be described in terms of a characteristic Damköhler number that is based on the ratio of a characteristic mixing time over a characteristic chemical time. As a fluid time, we can use the inverse of the scalar dissipation rate at the stoichiometric surface, $1 / \chi_{\mathrm{s}}$. The scalar dissipation rate can be defined in terms of the thermal diffusivity, $D_{\mathrm{T}}$, and of the thickness of the mixing layer, $\delta_{\mathrm{m}}$. Thus, $\chi_{\mathrm{s}} \approx D_{\mathrm{T}} / \delta_{\mathrm{m}}^{2}$ within a constant depending only on the flame overall stoichiometry. With $t_{\mathrm{L}}$ being the chemical time, the Damköhler number is defined as $D a=1 / \chi_{s} t_{\mathrm{L}}$. If the strain rate is changed with time by either modulating the velocity in one of the feed streams or by means of a vortex generated near the axis of the jet, as in the present experiments, $\delta_{\mathrm{m}}$ and, hence, $\chi_{\mathrm{s}}$ will also change with time. If $\chi_{\mathrm{s}}$ grows above the critical value required to extinguish the diffusion flame, that is, if $D a<D a_{\text {ext }}$, extinction occurs [26].

For a steady-state case, the thickness of the mixing layer satisfies $\delta_{\mathrm{m}}=\sqrt{D_{\mathrm{T}} / A}$. For a time-dependent problem, on the other hand, the thickness of the mixing layer must satisfy 


$$
\left\{\frac{1}{\delta_{\mathrm{m}}} \frac{d \delta_{\mathrm{m}}}{d t}+A\right\}=\frac{D_{\mathrm{T}}}{\delta_{\mathrm{m}}^{2}}
$$

where $A(t)$ is the time-dependent strain rate. Further details were presented in Ref. [27]. Equation 1 can be integrated using, for instance, $A=A_{0}$ for $t$ $<0$, when $\delta_{\mathrm{m}}=\delta_{\mathrm{m} 0}=\sqrt{D ; \mathrm{zT} / A_{0}}$ as the initial condition, leading to:

$$
\begin{aligned}
\left(\delta_{\mathrm{m}} / \delta_{\mathrm{m} 0}\right)^{2}= & \left.\left(1+2 \int_{0}^{t} \exp \int_{0}^{t^{\prime}} 2 A\left(t^{\prime \prime}\right) d t^{\prime \prime}\right) d t^{\prime}\right) \\
& \exp \left(-2 \int_{0}^{t} A\left(t^{\prime}\right) d t^{\prime}\right)
\end{aligned}
$$

This model is essentially equivalent to that originally introduced in Ref. [28], yielding an equivalent strain rate concept that was folded into a flamelet combustion model. Equation 2 shows that temporal changes in the strain rate, $A(t)$, will introduce changes in $\delta_{\mathrm{m}}(t)$ that will depend on the time history of the strain rate. These changes will also occur with a time-delay with respect to the onset of the perturbation. From the definition of $D a$, one concludes that the left-hand side of equation 2 can also be interpreted as the ratio of the instantaneous value of the Damköhler number, $D a=1 / \chi_{s} t_{\mathrm{L}}$, to its steadystate original value $D a_{0}$, that is,

$$
\left(\frac{\delta_{\mathrm{m}}}{\delta_{\mathrm{m} 0}}\right)^{2}=\left[\frac{1}{t_{\mathrm{L}}} \frac{\delta_{\mathrm{m}}^{2}}{D_{\mathrm{T}}}\right]\left[\frac{D_{\mathrm{T}}}{\delta_{\mathrm{m} 0}^{2}} t_{\mathrm{L}}\right]=\frac{D a}{D a_{0}}
$$

since, in the first approximation, $t_{\mathrm{L}}$ and $D_{\mathrm{T}}$ are not affected by the perturbation.

Equations 2 and 3 allow for the calculation of an effective scalar dissipation rate that is affected by the time history of the strain rate when a vortex approaches it, using as $A(t)$ the temporal profiles of the strain rate, as obtained experimentally, for example, as in Fig. 5. Introducing such a profile in the righthand side of Equation 2 and performing the integration numerically, we can compute the temporal evolution of $D a / D a_{0}$. In Fig. 5 , the calculated scalar dissipation rate from equation 2 is plotted versus time in addition to the already discussed experimental instantaneous strain rate. Clearly, the maximum computed scalar dissipation rate is smaller than the peak instantaneous strain rate, and a delay between the change of the strain rate and the scalar dissipation rate appears, as shown by the magnified scale in the inset of Fig. 5.

Next, we repeated the same procedure for all the experimental conditions of Fig. 6. The maximum non-dimensional scalar dissipation rate from equation 2 is plotted as a continuous line. It appears that the calculated values approach the quasi-steady extinction strain rates. The implication is that using the peak extinction strain rate to characterize the counterflow diffusion flames can be misleading under unsteady perturbations. Instead, it is preferable to cast the discussion in terms of scalar dissipation, which is the relevant quantity governing the flame burning rate up to extinction. We observe at differences larger than a factor of 2 between vortex-induced and quasi-steady extinction strain rates are reduced to no more than $30 \%$ in terms of scalar dissipation rate, or equivalently, $D a$ (for constant chemical time). Notice that the fact that the inner structure of the flame, the reactive-diffusive one, behaves quasi-steadily and is affected by the instantaneous value of $\chi_{\mathrm{s}}$ does not justify the direct adaptation of quasi-steady asymptotic analysis results to vortex-flame interaction experiments, as for example in Ref. [8]. In fact, the time history of the strain rate must be taken into account to arrive, via equation 2 , at the effective scalar dissipation rate felt by the flame.

\section{Implications for Turbulent Combustion}

The results reported here are of potential relevance to the flamelet models of turbulent combustion. The subject, in its simplest form, the so-called steady flamelet, has been extensively covered in a number of articles (e.g., [29]) without taking into account transient effects, under the assumption that they are rather infrequent and, as such, affect only the tail of the probability density function of the scalar dissipation rate. Yet, it can be argued that, especially under conditions of relatively large strain rate (and scalar dissipation rate), such as those encountered near the stabilization region of turbulent jet diffusion flames, these near-extinction conditions have a profound influence on the flame stabilization (e.g., [30]).

Transient effects were first taken into account in flamelet models in the pioneering work of Howarth et al. [28]. More recently, the so-called unsteady flamelet approach has been developed [31]. These authors modeled a turbulent jet flame and recognized the importance of transient effects in the anchoring region of such a flame in consideration of the rapid decay of the scalar dissipation rate along the axis of the flame and of the well-documented inability of steady flamelets to adjust to rapid changes. They then introduced unsteady effects with respect to a flamelet time, calculated from the jet nozzle, and examined the temporal evolution of the scalar dissipation as a function of such a time.

The following observations are in order. First, transient effects of this unsteady flamelet model account only for the rapid decay of the average scalar dissipation, but they do not account for fluctuations of the scalar dissipation rate. Second, a tabulation in terms of only $Z$ and $\chi_{\mathrm{s}}$ will be inadequate in representing the local instantaneous thermochemical state because it would not account for the time-delay effects discussed in connection with Fig. 5. These time-delay (or phase-lag) effects should be especially significant near extinction conditions when the flame 
exhibits the greatest sensitivity [18]. Third, time-history effects need to be accounted for to arrive at the appropriate definition of the local scalar dissipation rate and its fluctuations. This information is generally not provided by current engineering models of the turbulent fluid mechanics that are used in conjunction with the flamelet library. Thus, the findings of the present study are of direct relevance to future, more comprehensive transient flamelet models.

\section{Conclusions}

An experimental study was conducted on the extinction behavior of counterflow diffusion flames interacting with a vortex. Principal conclusions are as follows:

1. The difference between quasi-steady extinction and vortex-induced extinction was quantified experimentally.

2. Characteristic time estimates suggested that the flame reactive-diffusive inner zone behaves quasi-steadily, whereas the convective-diffusive outer layer is unsteady, as a result of the unsteady perturbation.

3. A simple time-dependent analytical model showed that the flame responds with a time delay to the perturbation, and extinction is reached when an effective scalar dissipation rate reaches a critical extinction value. This effective scalar dissipation rate depends on the time history of the strain rate. Consequently, despite the fact that the reactive-diffusive zone behaves quasisteadily, transient effects need to be accounted for to determine this effective scalar dissipation rate.

\section{Acknowledgments}

The authors wish to thank the following colleagues: Mr. Ernst Ebert for the design and construction of the vortex generation system and its retrofitting to the counterflow burner, Mr. Mikhail Noskov for preliminary calculations that enabled us to use $\mathrm{HCHO}$ as a flame marker, and, Dr. Adonios N. Karpetis for helping with aspects of the data acquisition. The support of NASA, under the Microgravity Science and Applications Program, Grant No. NAG3-1688 (Mr. Dennis Stocker, Contract Monitor), and of NSF, Grant No. CTS-9904296 (Dr. Farley Fisher, Contract Monitor) is gratefully acknowledged.

\section{REFERENCES}

1. Cuenot, B., and Poinsot, T., Proc. Combust. Inst. 25:1383-1390 (1994).

2. Marble, F. E., in Recent Advances in the Aerospace Sciences (C. Casci, ed.), Plenum, Amsterdam, Netherlands, 1985, p. 395.
3. Ashurts, W. T., and Williams, F. A., Proc. Combust. Inst. 23:543-550 (1990).

4. Roberts, W. L., and Driscoll, J. F., Combust. Flame 87:245-256 (1991).

5. Driscoll, J. F., Sutkus, D. J., Roberts, W. L., Post, M. E., and Goss, L. P., Combust. Sci. Technol. 96:213-229 (1994).

6. Rolon, J. C., Aguerre, F., and Candel, S., Combust. Flame 100:422-429 (1995).

7. Thevenin, D., Renard, P. H., Rolon, J. C., and Candel, S., Proc. Combust. Inst. 26:1079-1086 (1996).

8. Thevenin, D., Rolon, J. C., Renard, P. H., Kendrick, D. W., Veynante, D., and Candel, S., Proc. Combust. Inst. 27:719-726 (1998).

9. Renard, P. H., Rolon, J. C., Thevenin, D., and Candel, S., Combust. Flame 117:189-205 (1999).

10. Katta, V. R., Carter, C. D., Fiechtner, G. J., Roquermore, W. M., Gord, J. R., and Rolon, J. C., Proc. Combust. Inst. 27:587-594 (1998).

11. Saitoh, T., and Otsuka, Y., Combust. Sci. Technol. 12:135-146 (1976).

12. Darabiha, N., Combust. Sci. Technol. 86:163-181 (1992).

13. Ghoniem, A. F., Soteriou, M. C., Knio, O. M., and Cetagen, B., Proc. Combust. Inst. 24:223-230 (1992).

14. Barlow, R. S., and Chen, J. Y., Proc. Combust. Inst. 24:231-237 (1992).

15. Egolfopoulos, F. N., and Cambell, C. S., J. Fluid Mech. 318:1-29 (1996).

16. Im, H. G., Bechtold, J. K., and Law, C. K., Combust. Sci. Technol. 106:345-361 (1994).

17. Kim, J. S., and Williams, F. A., Combust. Flame 98:279-299 (1994).

18. Im, H. G., Law, C. K., Kim, J. S., and Williams, F. A., Combust. Flame 100:21-30 (1995).

19. Kistler, J. S., Sung, C. J., Kreutz, T. G., Law, C. K., and Nishioka, M., Proc. Combust. Inst. 26:113-120 (1996).

20. Brown, T. M., Pitz, R. W., and Sung, C. J., Proc. Combust. Inst. 27:703-710 (1998).

21. Im, H. G., Chen, J. H., and Chen, J. Y., Combust. Flame 118:204-212 (1999).

22. Seshadri K., Trevino, C., and Smooke, M. D., Combust. Flame 76:111-132 (1989).

23. Santoro, V. S., Kyritsis, D. C., and Gomez, A., "A Comparison of the Interaction of Laminar Vortices with either Gaseous or Spray Counterflow Diffusion Flames," the Joint Meeting of the US Sections of the Combustion Institute, Washington, DC, March 1517, 1999.

24. Seshadri, K., and Williams, F. A., Int. J. Heat Mass Transfer 21:251-253 (1978).

25. Liñán, A., and Williams, F. A., Combust. Flame 95:31-46 (1993).

26. Liñán, A., Acta Astronaut. 1:1007-1039 (1974).

27. Santoro, V. S., Liñán, A., and Gomez, A., "Unsteady Extinction Behavior of Counterflow Diffusion Flames: Experiments and Modeling," the Eastern States Section of the Combustion Institute, Raleigh, NC, October 10-13, 1999. 
28. Howarth, D. C., Drake, M. C., Pope, S. B., and Blint, R. J., Proc. Combust. Inst. 22:589-597 (1988).

29. Peters, N., Prog. Energy Combust. Sci. 10:319-339 (1984).
30. Takahashi, F., and Goss, L. P., Proc. Combust. Inst. 24:351-359 (1992).

31. Pitsch, H., Chan, M., and Peters, N., Proc. Combust. Inst. 27:1057-1064 (1998).

\section{COMMENTS}

Sébastien Candel, Ecole Centrale Paris, France. An alternative approach in dealing with unsteady flames is to use an effective strain rate. I was wondering if this might give a better match between your data and analysis. Also, would it have been possible to use a full non-steady onedimensional model together with the strain history to predict the extinction strain rate and compare it with your measurements?

Author's Reply.

1. The use of an effective strain rate, as originally introduced (Ref. [28] in this paper), is also possible. However, it is more fundamental to use an effective scalar dissipation. In fact, the square of the instantaneous stoichiometric scalar dissipation rate is proportional to the burning rate per unit flame surface, which defines the structure of the thin reaction layer. If the effective strain is defined as the one leading to the same effective scalar dissipation rate, the agreement between data and analysis would be the same as that presented in this paper. 2. The model presented in this paper, although qualitative, has the merit of highlighting the physics of these flames in a single revealing formula. Further numerical computations, with full chemistry and detailed calculation of the transport properties, are under way to reach a more quantitative agreement and to assess the importance of the kinetics on the extinction process. 